(c) 2011 IEEE. Personal use of this material is permitted. Permission from IEEE must be obtained for all other uses, in any current or future media, including reprinting/republishing this material for advertising or promotional purposes, creating new collective works, for resale or redistribution to servers or lists, or reuse of any copyrighted component of this work in other works. 


\title{
Using Meta-Networks to Identify Key Intervention Points in Nuclear WMD Development
}

\author{
W.H. Peiris, H. Armstrong, J. Venable \\ School of Information Systems \\ Curtin University of Technology \\ Perth, Western Australia \\ hasala@gmail.com, $\{$ h.armstrong, j.venable $\} @$ curtin.edu.au
}

\begin{abstract}
The ability to identify key intervention points in the nuclear WMD development process is vital for the development of effective intervention strategies against nuclear proliferation efforts. This paper describes research in progress to investigate nuclear weapons development as a meta-network of people, knowledge, resources, locations and tasks, and to design a software tool which will be capable of identifying the key intervention points of the process based upon the available information.
\end{abstract}

Keywords-nuclear WMD; key integrtion points; meta-network analysis, software tool.

\section{INTRODUCTION}

Military, regulatory and other organizations which attempt to disrupt the proliferation of nuclear weapons can deploy only limited resources, which must be used in an effective and efficient manner in order to cause maximum disruption or delay to the nuclear weapons programs. The effective and efficient use of resources usually requires them to be used against the most critical and vulnerable points of the nuclear weapons development process. Therefore, the identification of key intervention points of the nuclear WMD development process is vital for the formation of strategies for effective intervention.

However, the identification of key intervention points in the nuclear WMD development process is not a trivial task. Nuclear weapons development is a complex process which involves large numbers of people, tasks and resources[1]. Network science and especially meta-network modeling provides an ideal framework for the analysis of the nuclear WMD development process. A meta-network is a set of interconnected networks, which consists of multiple types of nodes (multimodal) and links (multiplex) [2, 3]. For example, a meta-network can consist of a social network (links among people), task network (links among tasks) and an assignment network (links between people and tasks). In the above example, the meta-network consists of three interconnected networks formed by two types of nodes (people and tasks).

This paper aims to describe research in its early stages to model nuclear weapons development as a meta-network and to design a software tool incorporating that model, which will be capable of identifying the key intervention points of the process based upon available information. The rest of this document gives an overview of prior research on the identification of critical intervention points of networks, a description of the proposed research project and a high-level overview of the proposed methodology for identifying key intervention points and evaluating relative merits of the available intervention options.

\section{OVERVIEW OF PRIOR RESEARCH}

\section{A. Use of Network Analysis Techniques}

With the advancement of theory and applications of network science, researchers have increasingly used such methods to identify critical entities, typically in social networks. Morstein and Perry [4] carried out Social Network Analysis (SNA) of the nuclear trading networks in order to detect potential nuclear WMD aspirants. They used three important measures of network centrality to identify key nations in both the supply and importer networks - degree, betweenness and closeness [4, 5]. In contrast to Morstein and Perry [4], Chen [6] has created a knowledge network of nuclear scientists and researchers in "high-risk" countries. He has used three techniques for his analysis - text mining to identify key scholars in the field using information published in the World Wide Web, Social Network Analysis to discover patterns of interaction between scholars, and visualization to discover conspicuous patterns in the network [6]. Lewis [7] has proposed a model-based vulnerability analysis (MBVA) method to identify vulnerabilities and quantitatively evaluate risks in critical infrastructure networks. The main advantage in Lewis' [7] method is that it provides a way of calculating optimal resource allocation for protecting critical infrastructure and means of prioritizing among several identified critical nodes or links. However, network analysis techniques described above typically use only one type of entity and one or two types of relationships. A meta-network modeling approach would allow a better representation of the domain.

\section{B. Meta-Network Analysis}

Although there is no prior evidence for the application of meta-network modeling to analyze the nuclear WMD development process, many other problems have been researched using similar techniques. For example, Carley et al. present their work on covert networks in two research papers on destabilizing networks [8] and destabilizing dynamic covert networks [2]. They argue that covert organizations are highly distributed and cellular in nature, thus presenting analysts who study them with the problem of an evolving, dynamic network in the presence of incomplete information [2]. Therefore, they 
have used Dynamic Network Analysis (DNA) techniques to analyze such networks [2]. Carley et al. have identified distinct types of entities present in a covert network - people, resources, knowledge and tasks [8] forming different types of networks using various entity combinations. According to the researchers, the DNA approach results in an evaluation of both network vulnerabilities and the impact of attacking the most vulnerable spots [9].

The techniques used by Carley et al. [2, 8] could be effective in the analysis of the process of nuclear weapons development. First of all, process of nuclear WMD development is a complex process, which can be modeled as a meta-network. Security analysts and policy advocates who analyze such networks often have to deal with uncertain, probabilistic information. Furthermore, proliferation networks are also dynamic in nature, adapting to various social pressures and opportunities. Although researchers such as Carley et al. [10] and Batagelj and Mrvar [11] have developed generic network analysis tools, none of them are customized to identify key intervention points in the nuclear WMD development process. Hutchins and Benham-Hutchins [12] successfully integrated generic network analysis software with other purpose-built tools to analyze criminal networks and a similar approach will be beneficial in the analysis of nuclear WMD meta-networks.

\section{Definition and Identification of Critical Nodes and Links}

Whenever the interest is in disrupting a network, as in the case of nuclear WMD production, researchers have focused on the identification of nodes whose removal would cause maximum fragmentation in the network. For example, Borgatti [13] defines critical nodes as a set of nodes whose removal would result in a network with least amount of cohesion. Similarly, Albert and Barabasi [14, 15] use two criteria to measure the criticality of nodes - extent of fragmentation caused by the removal of nodes and the increase in network diameter. However, most meta-networks are dynamic in nature and evolve in response to intervention[16]. Therefore, an initial fragmentation does not always guarantee disruption. Furthermore, the entire meta-network has to be considered when calculating the criticality of a node. Carley et al. [8] have defined additional criteria as indicators of network destabilization. These include the reduction in rate of flow of information through the network, reduced ability of the network to reach consensus as a decision making body and reduced effectiveness of the organization (for example, reduced accuracy of the tasks executed)[8].

Researchers [4, 6, 17-19] have used network analysis based on various metrics in order to identify critical nodes in networks. Some [4, 6] have used simple metrics based on centrality such as degree, betweenness and closeness [5]. Memon and Larson [18] have used structural analysis techniques based on cohesion, role and power (centrality) metrics in order to identify critical nodes in a network. Latora and Marchiori [17] have based their method of identifying critical nodes on a network-level metric called efficiency, which is an indication of how efficiently nodes in a network can exchange information. Borgatti [13] has pointed out two drawbacks of the use of typical centrality measures to identify critical nodes in networks called the goal issue and the ensemble issue. Carley et al. [2] have specified the problems of using metrics which depend only on the social network. First of all, such metrics can only find agents who are critical due to their ties in the social network but ignores the ones who are critical due to task they perform or resources they handle. Secondly, standard centrality metrics ignore the dynamic nature of networks. Carley et al. [2] have demonstrated the use of two meta-matrix based measures - cognitive load and task exclusivity and the results indicate that these metrics identify key entities which cannot be identified by standard social network analysis. In prior research [13, 17] critical links have been mainly identified by locating ones which optimize some network-level metric. However, in previous research, there is no evidence for the use of meta-network based metrics to locate critical links.

\section{RESEARCH METHODOLOGY}

\section{A. Design Science as the Research Paradigm}

Design Science Research (DSR) is research that develops new ways or technologies to solve generalized problems and make improvements. Rather than studying existing phenomena, it creates new phenomena to address generalized purposes. To do so, it designs and develops new artifacts [20,21], such as designs, models, methods, concepts that describe these, and instantiations of them. Since this research project is concerned with the creation of new artifacts - a meta-network model of proliferation of nuclear WMD, a design for an analysis tool, and a prototype instantiation of the analysis tool, design science $[20,21]$ is used as the research paradigm.

Venable's activity framework [22] is used as the research process model. The framework identifies four kinds of activities: theory building, solution technology invention, artificial evaluation, and naturalistic evaluation. The research steps in the next section follow the typical pattern of (1) theory building (in step 1 - postulating the suitability and high level design ideas for a solution to a problem), (2) solution technology invention (in steps 2-4 - developing the three artifacts mentioned in the previous paragraph), and (3) artificial evaluation (in step 5 - using focus groups and execution using historical data, but not actual use of a real system by real users in a real situation, which would be naturalistic evaluation). The omission of naturalistic evaluation is appropriate given the sensitive nature of the problem and difficulty in gaining access.

\section{B. Steps in the Research Project}

The research project will be carried out according to the following stages:

\section{1) Explore Past Research and Analyse Requirements}

The first stage of the project is to review past research on nuclear WMD development process, meta-network modeling and analysis tools. An analysis of the problem and functional requirements of the software tool will be also carried out in this phase. In order to identify the requirements of the analysis tool, opinions of intelligence analysts and subject domain experts will be sought.

\section{2) Analyse and Design the Meta-network Model}

The second stage involves the creation of a meta-network model to characterize the nuclear WMD development process. 
The meta-network of nuclear WMD development will consist of multiple classes of entities (nodes) and relationships (links). It will be based on the meta-matrix ontology proposed by Carley [23]. The following two tasks will be carried out at this stage:

\section{a) Investigate and Define the Meta-Network Ontology}

Prior research [1, 4, 6, 24] indicates that people, tasks, resources and knowledge are prerequisites for a nuclear weapons development project. Additionally, this research will consider locations as a type of entity since they are valuable as targets for intervention. The five selected entity types relate to the types of nodes that will be used to create the meta-network model. Preliminary analysis has identified that these entity types can create up to fifteen different networks due to various entity combinations (People $\mathrm{x}$ People, People $\mathrm{x}$ Tasks, Tasks $\mathrm{x}$ Tasks etc.). Each entity type combination can aslo have multiple types of relationships. However, when more than one relationship type exists between two entity types they will be aggregated. The contribution each entity type combination will provide will be explored and measured to identify networks that will generate the most useful information and insights. The attributes of the nodes must also be identified and this work is still under progress. Also, due to the uncertainty of available information, criteria must be developed to calculate the nature, weights and/or probabilities for links between the nodes.

\section{b) Create a Conceptual Meta-Network Model}

The meta-network model of the nuclear weapons development process can be depicted according to two layers of abstraction - a conceptual meta-network model and a metanetwork model populated with real data related to specific nuclear weapons projects. A conceptual meta-network model will use the five entity types - people, tasks, resources, knowledge and locations; as well as relationships between them to map the conceptual process of nuclear WMD development.

\section{3) Design the Software}

The design for a software tool will be generated based upon the meta-network model developed. A conceptual diagram depicting the planned design and operation of the analysis tool is given in Fig. 1. As indicated in the diagram, the analysis tool design will encapsulate six components. The user interface will accept commands and data related to the analysis of nuclear WMD projects and display the results of the analysis. Once data related to a specific nuclear WMD project is provided to the tool, it will analyze the data represented as a populated meta-network to identify critical nodes and links, or attributes of such that aid the analysis of key intervention points. Analysis algorithms needed for this will be incorporated as a separate module while the overall control, selection of metrics and measuring impact will be handled by the program control and heuristics module. After analyzing data the software tool will provide reports to the users. Additionally, there will be a visualization module to provide images of the meta-network, critical nodes and links. Since the analysis tool must be capable of identifying key intervention points and assessing the impact of intervention, the following tasks must be carried out under the design stage:

\section{a) Identify Strategies for Disruption}

There are many ways to disrupt a nuclear WMD metanetwork. Some possibilities include: removal of entities (removal of nodes), removal of relationships between entities (removal of links), change the nature, value or key attributes of nodes/links, and the introduction of new nodes or links. Out of these possibilities, the introduction of new nodes or links is a difficult task to achieve in a short time period. Carley [25] describe the unique problems associated with the introduction of new nodes and links and argue that removal of nodes is much more effective in destabilizing a network than the removal of links since removal of a node removes all links associated with the node. Modifying the nature, value or attributes of key nodes or links is another disruption strategy. Such modifications would make the completion of tasks in a nuclear WMD project mode difficult. Removal and/or modification of nodes and links require the identification of critical nodes and/or links as described in the next subsection.

\section{b) Identification of Critical Nodes and Links}

Out of the five node types of the Nuclear WMD metanetwork, tasks denote the work that needs to be done in order to produce nuclear WMD. Methods available for the production of nuclear WMD are not expected to change in a short time period and tasks will remain constant. Knowledge will be always related to either a person, resource or a task. Locations will house people and resources. Any intervention at a location will in turn affect people and resources related to that location. Therefore, there are two types of nodes which are candidates for intervention in a meta-network of nuclear WMD development: people and resources.

Various metrics have been developed to locate critical nodes in social networks. These include centrality based metrics such as degree, betweenness and closeness [5]. However, metrics such as cognitive demand and task exclusivity [2] can provide better insights on the critical agents in a meta-network. Therefore, both standard centrality based metrics and meta-network metrics will be used to locate key nodes in the meta-network. Furthermore, previous research indicates that the structure (i.e., topology) of a network is important in determining critical nodes [7, 8, 14-16]. Therefore, investigating the structure of the conceptual metanetwork will be useful to determine the appropriate metrics and algorithms that can be used to identify critical nodes. Besides the metrics already available, new meta-network metrics suitable for the analysis of nuclear WMD networks will be developed in this research and these will be used in combination with existing ones to locate critical nodes.

Identification of critical links will involve two tasks identifying types of links that can be removed or altered in nuclear WMD meta-networks and using appropriate metrics to locate critical links belonging to these types. For the purpose of locating the critical links, algorithms based on overall network fragmentation and increase in path lengths [13] will be used along with new meta-network based metrics to be developed in this research. 


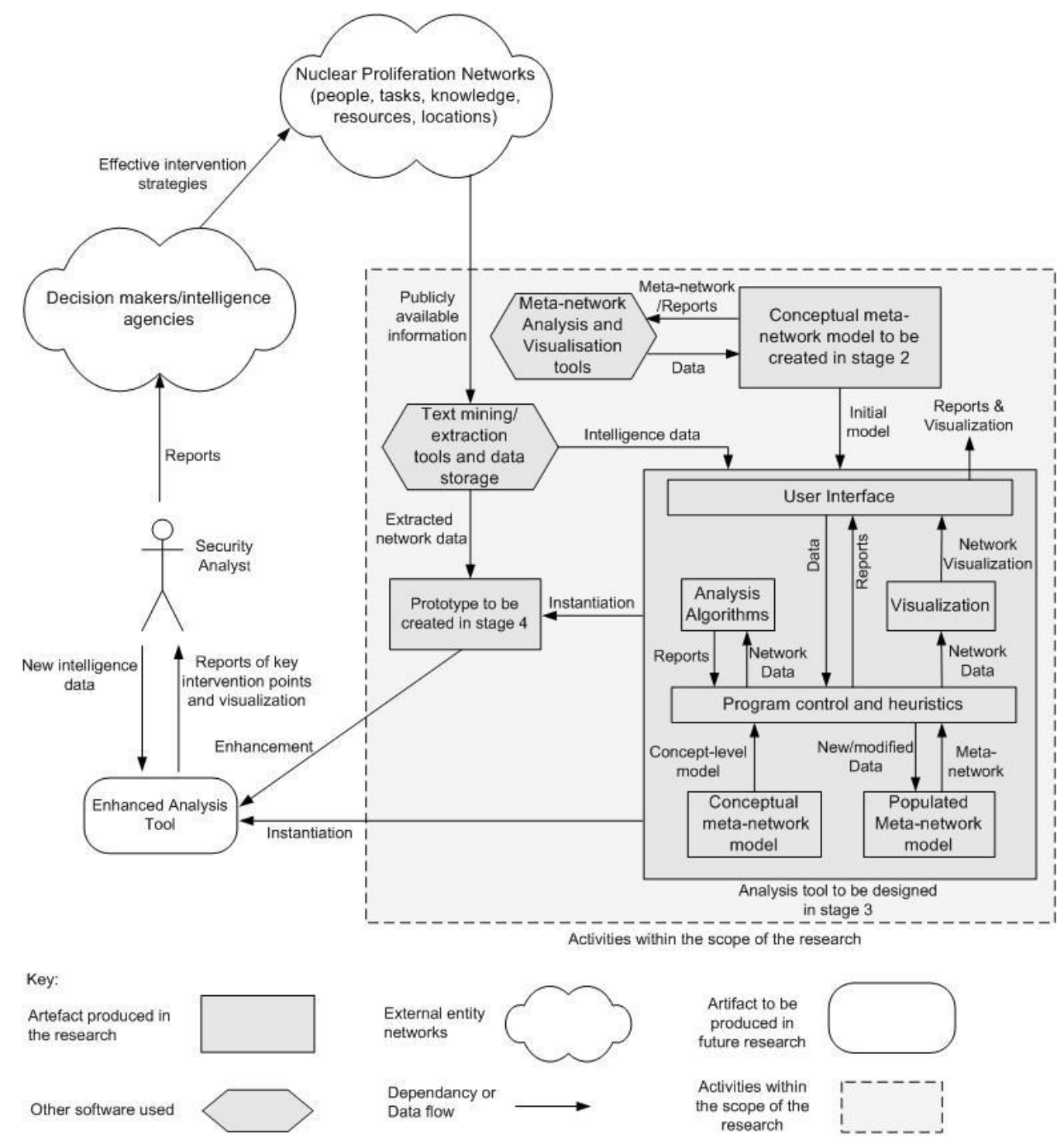

Figure 1. Conceptual design and operation of the analysis tool

\section{c) Define Criteria for Measuring the Expected Impact}

Most researchers have measured the disruption caused to a network in terms of the amount of fragmentation caused or the increase in path lengths [13-15]. However, nuclear WMD meta-networks consist of multiple, interconnected networks which would evolve in response to the intervention. Therefore, measuring disruption in dynamic meta-networks require a different set of criteria. Carley et al. [2, 8, 26] have developed a set of measures which include performance of the network, task accuracy and the rate of information diffusion. Although these measures can be used to gauge the disruption caused in nuclear WMD projects, ideally they should be supplemented by a set of new measures which focus on the task and project completion aspects.

Due to the dynamic nature of the nuclear WMD networks, it is important to look beyond the immediate consequences and estimate the potential impact of intervention over a period of time. Carley et al. $[2,16]$ have used multi-agent simulations to measure the impact of destabilization on terrorist and covert networks over a period of time. Comparable methods will be developed to measure the impact of intervention on the nuclear WMD meta-networks. For a given nuclear WMD project there will be multiple intervention options since intervention can be expanded to include a set of highly critical nodes or links. Furthermore, when removing or changing a group of nodes or links, the order of intervention can produce different results due to the dynamic nature of the meta-networks. Although knowing the relative impacts of many possible intervention options will be useful in decision making, there can be a large number of sequences generated even with a small set of critical nodes and links. Therefore, instead of attempting to find the best intervention sequence, the software tool will guide the analyst through the intervention options by providing what-if analysis capabilities.

\section{d) Compare Relative Merits of Intervention Strategies}

The tool will prompt the analyst to provide the nature of resources available for intervention. Once this information is provided the tool will use algorithms to deduce a suitable 
resource allocation strategy which maximizes the impact. Using results of the analysis, the tool will also provide a comparison of the relative impacts, cost and resource requirements of the potential intervention options. Graphs and tables will be used to present this information to the analyst.

\section{4) Develop a Software Prototype}

As proof of concept and to evaluate the design for the analysis tool, a prototype will be built. It will be built by adopting existing tools and applications whenever possible while adding extra functions or modules needed for the analysis of nuclear WMD development process. Evaluation of the prototype will be primarily used as a method of evaluating the design for the analysis tool. The prototype will be evaluated using a case study which analyzes actual data on Pakistan. Due to the large volume of available information, network text analysis tools will be used to extract data from publicly available sources. Diesner and Carley [27] and Carley et al. [10] have successfully carried out research projects in metanetwork analysis relying on publicly available information.

\section{DISCUSSION}

It is clear that meta-network analysis can be utilized to identify key intervention points in the nuclear WMD development process thereby eliminating most weaknesses in the prior research related to the topic. Research which has already been carried out in other problem domains, such as the identification of key individuals in terrorist and covert networks, provides a good basis for the analysis of the nuclear WMD development process and for estimating the potential impacts of interventions. However, the analysis of terrorist and covert networks takes a people-centric approach and there is a need to develop new metrics in order to analyze nuclear WMD meta-networks in a process-centric manner. This research is still at the problem/requirements analysis stage and the steps in the methodology are yet to be completed. The limitations of the research include the incapability to model the addition of nodes and/or links as an intervention strategy and the inability to carry out naturalistic evaluation. Furthermore, since the information on the nuclear WMD projects is likely to be incomplete and inaccurate, how erroneous data affects the results of the analysis must also be examined.

\section{REFERENCES}

[1] R. Harney, et al., "Anatomy of a Project to Produce a First Nuclear Weapon," Science \& Global Security: The Technical Basis for Arms Control, Disarmament, and Nonproliferation Initiatives, vol. 14, pp. 163 - 182, 2006.

[2] K. M. Carley, et al., "Destabilizing Dynamic Covert Networks," in Proceedings of the 8th International Command and Control Research and Technology Symposium, National Defense War College, Washington DC., 2003.

[3] I. Prestov, "Dynamic Network Analysis for Understanding Complex Systems and Processes," Defence Research and Development Canada. 2009.

[4] J. H. Morstein and W. D. Perry, "Commercial nuclear trading networks as indicators of nuclear weapons intentions," The Nonproliferation Review, vol. 7, pp. 75 - 91, 2000.

[5] S. Wasserman and K. Faust, Social Network Analysis: Methods and Applications. Cambridge: Cambridge University Press, 1994.
[6] H. Chen, "Nuclear Threat Detection Via the Nuclear Web and Dark Web: Framework and Preliminary Study," in Intelligence and Security Informatics. vol. 5376, D. Ortiz-Arroyo, et al., Eds., ed: Springer Berlin / Heidelberg, 2008, pp. 85-96.

[7] T. G. Lewis, Critical infrastructure protection in homeland security: defending a networked nation. Hoboken, New Jersey.: John Wiley and Sons, 2006.

[8] K. M. Carley, et al., "Destabilizing Networks," Connections, vol. 24, pp. 79-92, 2001.

[9] K. M. Carley, et al., "Destabilizing terrorist networks," in North American Association for Computational Social and Organizational Science Conference, Pittsburgh, Pennsylvania, 2003.

[10] K. M. Carley, et al., "Toward an interoperable dynamic network analysis toolkit," Decision Support Systems, vol. 43, pp. 1324-1347, 2007.

[11] V. Batagelj and A. Mrvar, "Pajek - Analysis and Visualization of Large Networks," in Graph Drawing. vol. 2265/2002, ed Berlin: Springer Berlin / Heidelberg, 2002, pp. 8-11.

[12] C. Hutchins and M. Benham-Hutchins, "Hiding in plain sight: criminal network analysis," Computational \& Mathematical Organization Theory, 2009.

[13] S. Borgatti, "Identifying sets of key players in a social network," Computational and Mathematical Organization Theory, vol. 12, pp. 21 34, 2006.

[14] R. Albert and A. L. Barabási, "Statistical mechanics of complex networks," Reviews of modern physics, vol. 74, pp. 47-97, 2002.

[15] R. Albert, et al., "Error and attack tolerance of complex networks," Nature, vol. 406, pp. 378-382, 2000.

[16] K. M. Carley, "Dynamic network analysis," Comittee on Human Factors, National Research Council Washington, DC2003.

[17] V. Latora and M. Marchiori, "How the science of complex networks can help developing strategies against terrorism," Chaos, solitons \& fractals, vol. 20, pp. 69-75, 2004.

[18] N. Memon and H. L. Larsen, "Structural analysis and destabilizing terrorist networks," in International Conference on Data Mining (DMIN 2006), Las Vegas, Nevada., 2006, pp. 296-302.

[19] N. Memon and H. L. Larsen, "Practical approaches for analysis, visualization and destabilizing terrorist networks," in Availability, Reliability and Security, 2006. ARES 2006. The First International Conference on, Esbjerg, Denmark, 2006, p. 8 pp.

[20] A. Hevner and S. Chatterjee, "Design Science Research in Information Systems," in Design Research in Information Systems. vol. 22, ed: Springer US, 2010, pp. 9-22.

[21] S. T. March and G. F. Smith, "Design and natural science research on information technology," Decision Support Systems, vol. 15, pp. 251266, 1995.

[22] J. Venable, "A framework for Design Science research activities," in 2006 Information Resource Management Association Conference, Washington, DC, USA, 2006.

[23] K. M. Carley, "Smart Agents and Organizations of the Future," in Handbook of New Media: Social Shaping and Consequences of ICTs, L. A.L. and L. S.M., Eds., ed London: SAGE, 2002.

[24] D. MacKenzie and G. Spinardi, "Tacit Knowledge, Weapons Design, and the Uninvention of Nuclear Weapons," The American Journal of Sociology, vol. 101, pp. 44-99, 1995.

[25] K. M. Carley, "Inhibiting Adaptation," in Command and Control Research and Technology Symposium, Navel Postgraduate School, Monterey, CA, 2002.

[26] K. M. Carley, "Estimating Vulnerabilities in Large Covert Networks," in 9th International Command and Control Research and Technology Symposium, Vienna, VA, 2004.

[27] J. Diesner and K. M. Carley, "Using Network Text Analysis to Detect the Organizational Structure of Covert Networks," in Proceedings of the NAACSOS 2004 Conference, Pittsburgh, PA, 2004. 